\title{
Os usos de um aplicativo de saúde móvel e a educação dos corpos em uma política pública
}

\author{
Leonardo Trápaga Abib ${ }^{\circledR} \odot$, Ivan Marcelo Gomes ${ }^{\mathrm{a}}$, Eduardo Lautaro Galak ${ }^{\mathrm{b}} \odot$
}

\section{Palavras Chave:}

Saúde móvel; Políticas públicas; Atividade física; Prevenção em saúde.

\section{Keywords:}

Mobile health;

Public polices; Phisycal activity; Health prevention.

\begin{abstract}
RESUMO
No Estado do Espírito Santo a Secretaria de Estado da Saúde criou o aplicativo de saúde móvel "Movimento 21 dias por uma vida mais saudável" (M21) para estimular a população a ter hábitos considerados mais saudáveis. O objetivo deste estudo foi apresentar e discutir, através das postagens nas redes sociais, os modos pelos quais alguns dos participantes do M21 buscaram relacionar-se com o programa. A principal estratégia de pesquisa utilizada foi o mapeamento da \#Movimento21dias nas redes sociais em um período de dois anos (2016-2018). A partir dos dados oriundos da página do M21 e das postagens dos usuários em redes sociais, notou-se que o programa teve baixa adesão e notoriedade. Em relação os usos, conclui-se que os sujeitos mobilizam modos singulares para lidar com os discursos do M21, manifestando movimentos híbridos e distintos.
\end{abstract}

\begin{abstract}
In the state of Espírito Santo, the State Department of Health created the mobile health application "Movimento 21 dias por uma vida mais saudável" (M21) to encourage the population to have healthier habits. The aim of this study was to present and discuss, through posts on social networks, the ways in which some of the participants of M21 sought to relate to the program. The main research strategy used was the mapping of \#Movimento21dias in social networks over a period of two years (2016-2018). From the data coming from the M21 page and the user's posts on social networks, it was noted that the program had low adherence and notoriety. Regarding the uses, it is concluded that the subjects mobilize singular ways to deal with the discourses of M21, manifesting hybrid and distinct movements.
\end{abstract}

\section{RESUMEN}

Salud asistida por móvil; Políticas públicas; Actividades Físicas; Prevención de la salud.

Mediante la creación del aplicativo móvil "Movimento 21 dias por uma vida mais saudável” (M21) por parte de la Secretaría de Salud del Estado de Espírito Santo en Brasil se propuso estimular la población para tener hábitos considerados como saludables. El objetivo de este estudio fue presentar y discutir, a través de publicaciones en redes sociales, las formas en que algunos de los participantes de M21 intentaron relacionarse con el programa. La principal estrategia de investigación utilizada fue el mapeamento del hashtag \#Movimento21dias en las redes sociales durante un período de dos años, entre 2016 y 2018. A partir de los datos obtenidos de la página web del M21 y de los posteos de los usuarios en las redes sociales, se observó que el programa tuvo una baja adhesión y notoriedad. En relación con los usos, se concluye que los sujetos movilizan modos singulares para lidiar con los discursos del M21, manifestando movimientos híbridos y distintos.

\footnotetext{
a Universidade Federal do Espírito Santo, Vitória, ES, Brasil.

b Universidad Nacional de La Plata, Facultad de Humanidades y Ciencias de la Educación, Centro Interdisciplinario Cuerpo, Educación y Sociedad, La Plata, Argentina.
}

Autor correspondente: Leonardo Trápaga Abib

E-mail: leoabib@gmail.com

Recebido em 6 de Novembro de 2019; Aceito em 3 de Março de 2020. 


\section{CONSIDERAÇÕES INICIAIS}

Com o advento das novas tecnologias da informação, da expansão do acesso à internet e do aumento do consumo de dispositivos móveis, como celulares e tablets, campanhas e programas de educação e promoção da saúde tem sido incorporadas no campo da mHealth (Riley et al., 2011). A mHealth (saúde móvel) consiste no uso de tecnologias de computação e comunicação móveis para cuidados pessoais de saúde, para uso de instituições privadas e para programas e ações de saúde pública (Who, 2011). Os principais exemplos de serviços de mHealth são os sites e aplicativos para serem usados em dispositivos móveis. Atualmente, segundo Matravolgyi (2016), existem mais de 165 mil aplicativos voltados para a área da saúde, sendo utilizados para variadas finalidades, como por exemplo, coletar dados referentes à glicemia, peso, pressão arterial, níveis de sono e de estresse, quantidade de calorias ingeridas e gastadas em atividades físicas, entre outros. Além dessas, outra característica cada vez mais comum no universo dos dispositivos de saúde móvel é a difusão de aplicativos de educação em saúde com foco na prevenção a partir da difusão de informações sobre estilo de vida saudável (Rocha et al., 2016).

No Brasil, cresce o número de agências públicas e privadas que utilizam aplicativos de $m$ Health para desenvolverem campanhas e ações em saúde. O Estado do Espírito Santo endossa essa realidade desde o ano de 2015, quando o governo estadual lançou, através da sua Secretaria de Estado da Saúde (SESA), em parceria com uma agência de publicidade ${ }^{1}$, o programa Movimento 21 dias por uma vida mais saudável (M21). O objetivo do programa é fornecer por meio de um site ${ }^{2}$ e de um aplicativo para dispositivos móveis, informações e conselhos sobre convívio familiar, alimentação saudável e atividade física. O nome Movimento 21 dias dá-se pelo fato de que os idealizadores do programa se valem da tese que ao praticar uma mesma ação por 21 dias consecutivos ela pode tornar-se um hábito na vida da pessoa, fazendo com que ela a incorpore com mais facilidade em sua rotina. As bases mencionadas por um dos criadores do programa (vinculado à uma agência de publicidade) para justificar tal escolha são as de trabalhos da linha comportamental, como os de Maxwell Maltz - autor que buscou sustentar o argumento de que 21 dias seria o tempo mínimo para se aprender um novo hábito -, John Hargravee, entre 1 A respectiva agência havia sido aprovada em um dos editais do Governo do Estado. Após ser contemplada pelo edital, a agência foi indicada para a SESA, que a designou para, entre outras tarefas, criar uma campanha na área da promoção de saúde.

20 endereço do site do Movimento 21 dias por uma vida mais saudável é < https://www.movimento21dias.com.br/> outros. Por outro lado, as referências mobilizadas pelas servidoras da SESA para elaboração do programa, são oriundas da Política Nacional de Promoção da Saúde (Brasil, 2006), dos dados epidemiológicos produzidos pela própria secretaria ${ }^{3}$.

Portanto, a ideia central do M21 é estimular nos seus usuários o interesse em práticas consideradas importantes para construção de um estilo de vida saudável. As atividades propostas pelo M21 são bastante variadas. Por exemplo, no grupo da alimentação saudável, constam desafios como baixar o consumo de açúcar, sal e produtos industrializados, ingerir mais frutas, legumes e verduras, comer de 3 em 3 horas, entre outros. Já no grupo das atividades físicas, alguns dos desafios sugeridos são subir/descer escadas, caminhar/correr por 30 minutos, alongar-se durante o dia, andar de bicicleta entre outros. Por fim, no grupo do convívio familiar surgem desafios como reunir a família à mesa nas refeições, brincar ou jogar em família e criar projetos familiares. Os desafios da alimentação e do convívio familiar presentes no M21 foram produzidos por servidores da SESA ligados as áreas da nutrição e psicologia, enquanto que os desafios da atividade física ficaram a cargo de professores de educação física vinculados a outra instituição pública (Abib, 2019).

Até a data de 15 de outubro de 2018, segundo dados fornecidos pelo próprio site do Movimento 21 dias, havia cadastrados 32.936 usuários. Ainda de acordo com o programa, as cidades que possuem mais sujeitos cadastrados foram as da região metropolitana da Grande Vitória. Desses 32.936 cadastrados, apenas 894 (2,8\%) relataram ao aplicativo/site terem concluído ao menos um desafio (ou seja, praticar a mesma ação por 21 dias consecutivos). Ao levar em consideração o número de pessoas que fizeram pelo menos 1 dos 21 dias do seu desafio escolhido, o número passa a ser de 9.074 indivíduos.

De modo a compreender de maneira mais ampla o espectro do Movimento 21 dias. analisamos os diversos conteúdos presentes em suas plataformas digitais. Nesse sentido, identificou-se uma ênfase maior no uso de enunciados e discursos pautados pelas teses preventivistas e comportamentalistas, mobilizando estratégias disciplinares, biopedagógicas e pastorais (Foucault, 2008; Wright e Harwood, 2009), como o incentivo à práticas regulatórias de automonitoramento por parte dos sujeitos. O estímulo ao governo de si e à gestão indivividual dos riscos e comportamentos é outra marca recorrente nos textos, vídeos e imagens

3 Uma análise mais aprofundada em relação às referências e ao processo de construção do programa pode ser encontrada na tese de doutorado (Abib, 2019) que embasa o presente artigo. 
presentes no aplicativo e no site do programa (Abib e Gomes, 2019).

Já em um primeiro exercício de observação de postagens em redes sociais encontramos uma série de postagens de usuários do M21 compartilhando suas experiências, dificuldades e êxitos quanto à prática dos desafios elaborados pelo programa, sendo a atividade física um eixo recorrente nessas postagens. Diante desse cenário optamos por aprofundar a investigação acerca das postagens com o objetivo de analisar algumas das possibilidades de usos do M21 segundo os relatos e experiências compartilhadas nas redes sociais por parte dos usuários do programa. Dessa forma dedicamo-nos a apresentar e discutir, através das postagens nas redes sociais, os modos pelos quais alguns dos participantes do Movimento 21 dias buscaram relacionar-se com o programa. Ademais, buscou-se compreender como que os indivíduos podem vir a construir sentidos aos discursos sobre saúde e estilo de vida difundidos pelo M21.

\section{METODOLOGIA}

Nas plataformas digitais do Movimento 21 dias são disponibilizados notícias, dados estatísticos, informações científicas sobre temas relacionados à saúde e estilo de vida, além das dicas e dos desafios. Ao realizar gratuitamente o download do aplicativo, os usuários passam a receber mensagens, alertas e avisos do programa. O M21 também comunica-se com seus usuários via aplicativo através do envio de mensagens de incentivo e congratulação pela realização dos desafios. Por outro lado, nos casos em que os sujeitos passam muito tempo sem acessar o aplicativo e realizar os desafios, o M21 envia email's para encorajar os usuários a voltarem para o programa ${ }^{4}$.

Tanto no site quanto no aplicativo, o M21 incentiva seus usuários cadastrados a utilizarem suas redes sociais para compartilharem fotos e mensagens sobre o andamento de seus desafios inserindo a hashtag \#Movimento21dias como uma forma de obter algum retorno por parte dos participantes além de promover o programa e incentivar mais pessoas a aderirem ao programa.

Enquanto um programa que se vale das plataformas digitais para difundir conselhos e informações sobre prevenção em saúde e adoção de hábitos saudáveis, optamos primeiramente por investigar o conteúdo presente no site $e^{5}$ do Movimento 21 dias, tal qual sugerem

4 Outra forma de comunicar-se com a população foi através de palestras com representantes dos municípios capixabas, setores do Governo Estadual e entidades privadas, além também de ações realizadas nos terminais de ônibus da região metropolitana da grande Vitória-ES.

5 A partir do endereço <http://www.movimento21dias.com.br/> outras produções sobre dispositivos de saúde móvel (Lupton e Thomas, 2015; Fullagar et al., 2017). Num segundo momento, a exemplo do trabalho de Fotopoulou e O'Riordan (2016), realizamos o download do aplicativo do M21 para um celular pessoal, com o intuito de entender como era fazer parte dessa rede, como ela funcionava, como se dava a comunicação com os usuários, quais tipos de mensagens eram enviadas, etc.

Já a estratégia metodológica produzida durante o percurso de pesquisa para investigar as relações estabelecidas entre usuários e programa foi o mapeamento do mesmo nas redes sociais Facebook, Twitter e Instagram. Tendo em vista que: i) o próprio M21 incentiva seus usuários a compartilharem informações sobre as escolhas e o andamento dos desafios e, ii) a expansão do campo de pesquisas e estudos sobre monitoramento de mídias sociais (Fragoso et al., 2011); decidimos realizar uma busca das publicações sobre o M21 nas redes sociais e, assim, produzir um banco de imagens e postagens obtidas através dessa ação, constituindo-se em nosso material para análise.

Para realizar tal tarefa investigativa seguimos as pistas das pesquisas com redes sociais (Goodyear, 2017; Silva e Stabile, 2016) e adotamos os seguintes passos para realização do presente estudo: primeiro, foi utilizada a hashtag ${ }^{6}$ (\#) ligada ao programa - no caso, \#Movimento21dias - como termo chave no módulo de busca das redes sociais Facebook, Twitter e Instagram; segundo, foram analisadas somente as publicações feitas no "modo público" (as quais qualquer usuário das redes pode ver); terceiro, conferiu-se se a publicação estava realmente falando sobre o programa M21; por fim, após essas etapas, produziu-se um banco de dados contendo as publicações (imagem e texto) extraídas das redes sociais ${ }^{7}$ e com isso foram feitas as análises de como os usuários cadastrados no M21 relacionam-se com o respectivo programa. Dentre as limitações observados ao largo do estudo, pontuamos a dificuldade para acompanhar de maneira mais contínua e presencial os usuários do M21 - devido às vicissitudes em acessá-los para construir espaços e canais de diálogos - e o fato de somente podermos analisar as postagens feitas por contas públicas, 0

foi possível acessar todos os materiais utilizados para esta pesquisa, notícias, desafios, dicas, imagens, vídeos, peças gráficas, o ranking dos usuários e algumas das estatísticas do programa.

6 Quando um usuário de redes sociais adiciona uma hashtag à sua postagem, ela passa a ser indexada pelo site (Facebook, Twitter, Instagram, etc.) e torna-se pesquisável pelos demais usuários. Ao clicar na hashtag, a pessoa é direcionada para uma página que lista todas as postagens realizadas com as mesmas hashtags.

7 O período da coleta de dados oriundos das redes sociais deu-se de outubro de 2016 até outubro de 2018 
que restringiu o quantitativo de sujeitos investigados (Abib, 2019).

Os resultados e análises desta investigação estão sistematizados em dois eixos: no primeiro discutiremos acerca das práticas de automonitoramento e de consumo realizadas e visibilizadas pelos usuários analisados; na segunda trazemos à tona o elemento conselheiro que algumas das postagens analisadas adquirem, bem como o caráter relacional da saúde que é apontado por parte dos sujeitos através de suas mensagens compartilhadas nas redes. Apesar de estarem divididos, os eixos articulam-se entre si de maneira complementar de modo a dar visibilidade à complexidade das relações que são estabelecidas entre sujeitos e o programa Movimento 21 dias.

\section{Práticas de selftracking e consumo}

Nesta seção serão tratadas as formas pelas quais os sujeitosanalisadosforamadministrandoeincorporando o ideário da vida saudável em seus cotidianos, levando em consideração que nessa administração do eu contemporâneo, "[...] as capacidades pessoais e subjetivas dos cidadãos têm sido incorporadas aos objetivos e aspirações dos poderes públicos" (Rose, 1998, p. 32) constituindo, assim, relações com as políticas de instituições públicas e privadas. Diante disso, a partir do mapeamento, da análise e interpretação de postagens de usuários do M21 nas rede sociais, foram produzidas algumas reflexões sobre como os sujeitos convertem todas essas informações e conselhos fornecidos pelo programa para a produção de suas "técnicas do eu" (Rose, 1998), criando, assim, suas próprias estratégias de autocuidado, via dietas, exercícios físicos e momentos em família.

Enquanto um confessionário moderno, as redes sociais tem constituído-se em espaços onde muitos sujeitos trazem à tona uma série de relatos sobre cotidiano, sobre suas dificuldades, seus "pecados", suas conquistas, vitórias, aprendizados, etc. Por isso, a partir do acompanhamento das postagens contendo a \#Movimento21dias, foi possível refletir acerca das estratégias de cuidado e selftracking ${ }^{8}$ praticadas pelos usuários do M21. Nessas muitas "confissões" compartilhadas em público, encontramos distintas manifestações, sensações, experiências, assim como diferentes

8 Utilizamos o termo em inglês selftracking por ser o mais referenciado na literatura sobre a temática. No Brasil, esse termo foi traduzido por Castiel, Moraes e Paula (2016) para "automonitoramento". perfis pessoais e institucionais. A partir disso ficou notório o quanto algumas pessoas estão implicadas em publicizar suas rotinas, e, neste caso, em contribuir para o endosso das práticas de selftracking promovidas pelos dispositivos de saúde móvel (Lupton, 2018). Abaixo, estão expostos alguns exemplos de usuários $^{9}$ do Movimento 21 dias que costumam compartilhar mensagens a respeito dos desafios que elegeram para praticar:

'Daqui a um ano você vai ter desejado começar hoje': foi assim que fui convencida a aceitar o desafio da Secretaria Estadual de Saúde para aderir ao Movimento 21 Dias por uma vida mais saudável. Escolhi alimentação saudável e atividades físicas [...] E que Deus me ajude a ficar longe dos doces! \#movimento21dias \#projetoverãoparte50 (Usuária 1 do Instagram). Meu primeiro dia do \#movimento21dias... Corpo, mente e espírito igualmente cuidados. Comecei com minha sessão de oração, dedicação, caminhada e abdominais. Depois disso uma praia pra relaxar a mente e ficar mais pertinho da natureza de Deus [...] Estou comendo em porções menores, evitando carboidratos a partir das 19:00h e evitando também o açúcar (ainda não consegui eliminar de vez e nem sei se quero eliminar). Não gosto de muita restrição (Usuária 1 do Facebook).

Menções a uma promessa de um futuro melhor são recorrentes em agumas postagens, mostrando que o caráter preventivista ao qual o Movimento 21 dias apega-se é um dos elementos que motivaram as pessoas a aderirem ao programa. Nesse caso, as práticas de selftracking mais utilizadas por dispositivos da área da prevenção e promoção da saúde podem ser consideradas como parte das estratégias e dos "discursos heterogêneos que colocam o 'si mesmo liberal' como um cidadão responsável, com vontade e capacidade de tomar cuidado de si ou de seus autointeresses e bem-estar" (Castiel et al., 2016, p. 104). Para Deborah Lupton (2012), o discurso da promoção da saúde, quando difundido por dispositivos de saúde móvel representa os indivíduos enquanto atores dispostos a assumirem as responsabilidades de promoverem a sua própria saúde. A respeito dos comportamentos individuais, o "combate" às

9 Nesta pesquisa optou-se em manter o anonimato dos usários que publicaram postagens sobre o Movimento 21 dias. Na literatura revisada acerca de pesquisas com imagens de redes sociais, não encontrou-se um consenso quanto a qual seria a melhor maneira de expô-las. Para este artigo, optou-se por trazer somente os textos sem mostrar os rostos das pessoas. 
tentações, a resistência e à preguiça e o relato da sensação de responsabilidade sobre si também são marcas recorrentes nas postagens dos usuários do M21:

Hoje tá sendo um dia difícil de resistir a tentação de comer besteira, a geladeira do serviço está cheia de guloseimas e uma torta de morango. Bom tô fingindo que não tô vendo e fazendo refeição normal (Usuária 2 do Facebook).

Confesso que a preguiça era monstra fui quase de olhos fechados, mas cumpri mais 1 dia do desafio (Usuário 1 do Twitter).

Eu aderi! Porque promover mudanças de hábitos por pelo menos 21 dias seguidos ajudam a fazer com que eles se mantenham depois em nossa vida. Porque tava precisando recuperar a vergonha na cara e o rítmo pra retomar a eliminação de uns últimos e insistentes quilos. Já tô no meu \#dia3 e a minha maior mudança é fazer atividade física necessariamente todos os dias nesses 21 dias (Usuária 2 do Instagram).

A pressão moral sobre o próprio comportamento é tema recorrente nas mensagens escritas e compartilhadas pelos usuários do Movimento 21 dias. O uso de expressões "vergonha na cara", "confesso que falhei bastante", "tive medo de fracassar", "a preguiça era monstra", "tá difícil resistir a tanta besteira" demonstram como e o quanto a lógica da individualização da responsabilidade pode ter efeitos sobre a subjetividade e a construção de identidades pessoais. De acordo com Lupton (2012), as novas tecnologias na área da saúde, como os dispositivos de $m$ Health, podem contribuir para a produção de sentimentos de vergonha e culpa em seus usuáriosalvo. Byung-Chul Han (2014) argumenta que a questão do fracasso dentro de uma sociedade orientada pela racionalidade neoliberal, muito voltada para a competição e o rendimento, resulta em um indivíduo que se culpa e sente vergonha de si mesmo ao invés de, por exemplo, pôr em dúvida a sociedade ou o sistema político e econômico em que vive.

Já em relação às práticas de consumo relacionadas ao estilo de vida saudável, pode-se notar, a partir da visualização das imagens e postagens compartilhadas em rede social, que os usuários do M21 apresentam uma gama de materiais cujo uso costuma ser incentivado pelo vasto e emergente mercado da vida ativa (Fraga, 2006). Bicicletas bem equipadas, roupas e calçados próprios para atividade física e ingredientes refinados aparecem com alguma frequência. Quanto aos espaços e equipamentos mais visualizados nessas postagens, nota-se que há também uma mescla entre usuários que realizam atividades tanto em espaços privados, principalmente academias de musculação, e usuários que praticam seus desafios em espaços públicos, como a praia:

Dia de perna \#diadodesafio \#movimento21dias \#mundobt \#bt - em Academia BodyTech (Usuária 3 do Facebook).

Acordei bem cedinho hoje para começar a cumprir mais uma meta para 2017: voltar a fazer exercício físico. Ficou ainda mais fácil com a companhia da minha querida vizinha \#Xôsedentarismo \#xôgordices \#movimento21dias \#vidasaudável (Usuária 4 do Facebook).

Porque dia de sábado é dia de correr e caminhar. Eu tenho um objetivo e uma meta pra cumprir \#movimento21dias \#semenrolação \#éprafrentequeseanda \#menos $12 \mathrm{~kg}$ (Usuária 5 do Facebook).

Os modos pelos quais os usuários analisados relacionam-se com os conselhos e informações do Movimento 21 dias permite-nos pensar o quanto a estetização da vida (Ravettino, 2008) ajuda a constituir a identidade desses sujeitos. Aliar mensagens, hashtags e imagens dentro de academias e parques que demarquem um determinado estilo de vida mais vinculado à saúde e à família é uma marca constante e emergente nas redes sociais, o que indica nesse processo de estetização da vida cotidiana uma necessidade de apresentar-se como parte de algo, de alguma tribo.

\section{Novos conselheiros e práticas relacionais em saúde}

Dentro do bojo das "técnicas do eu" (Rose, 1998) acionadas pelos sujeitos há espaço para práticas de automonitoramento, cobrança de si e valorização do orgânico em detrimento do social, há, por outro lado, também, abertura para outras práticas, de caráter mais relacional e de construção de redes de apoio e incentivo. Tais práticas, embora ambivalentes, constituem o rol de alternativas dos usuários do M21 analisados, como nos indicam os compartilhamentos dos mesmos em seus perfis pessoais nas redes sociais. Portanto, para além dos sentimentos e sensações de autocobrança, os usuários também mostraram outras formas de se relacionarem com o programa, chegando inclusive a se constituirem em multiplicadores do M21 e conselheiros da vida saudável (Gomes, 2008):

Feriado que fala né?? Hoje rolou aquele cardio de 40min, inclusive esse maravilhoso exercício 'bike abdominal em 2 tempos'. Fique ligado nos stories para acompanhar nossa rotina de exercícios e nossa alimentação. \#elasnarua 
\#vemcomagente\#movimento21dias

\#treinoemcasa\#alimentacaosaudavel

\#tododiaédia\#foco\#vemresultado

\#vidasaudavel (Usuária 3 do Instagram).

Hoje dei início ao \#movimento21dias em busca

de adotar hábitos mais saudáveis e uma vida

mais plena [...] Pra você que deseja realizar alguma atividade física mas tá com orçamento apertado ou mesmo sem tempo indico o canal 'Exercício em casa' no YouTube. Lá você pode encontrar diversas videoaulas e se exercitar sem ter que sair da sala (Usuário 6 do Facebook).

Como podemos observar nas postagens acima, as pessoas, além de anunciarem o que estão fazendo, convidam abertamente seus seguidores para que participem do Movimento 21 dias, colocando o nome do aplicativo e links sobre o programa nas publicações. Com isso, os usuários podem ir construindo uma rede mútua de apoio a fim de incentivar cada um a seguir seus desafios em busca de uma melhor situação de saúde. Aqui alguns usuários inclusive explicam sua rotina de exercícios e sugerem a seus seguidores algumas atividades para que sejam incorporadas por eles em suas rotinas, mostrando como utilizam diferentes tipos de conhecimentos para si ao ponto de tornarem-se também conselheiros da vida saudável (Gomes, 2008).

Como abordado na seção anterior, a partir da emergência do processo de estetização da vida que implica, entre outras coisas, no compartilhamento de imagens e mensagens do cotidiano pessoal, é interessante interpretar nas postagens trazidas até o momento aqueles elementos relacionados a percepções pessoais como, engajamento, fé, motivação, estar em família, com amigos, com a natureza. ou seja, são demonstrações de aspectos que vão além, por exemplo, das prescrições médicas ou de outras autoridades profissionais, científicas e midiáticas:

Família fitness pedala junto! \#movimento21dias Família unida para pedalar (Usuária 7 do Facebook).

Das maravilhas de acordar cedo e de saber aproveitar o que a vida tem para nos oferecer [...] \#nadaecorre \#CriaTrabalhaeConfia \#amores \#movimento21dias (Usuária 8 do Facebook).

De que adianta trabalhar tanto, estudar tanto e ver sua saúde indo embora? \#movimento21dias \#correndoatrásdoprejuizo \#boracaminhar \#umpoucodesol (Usuária 9 do Facebook).

Quando você começa a atrair aquilo que você focou e a receber de volta toda a energia positiva que emitiu. O que fazer? Agradecer \#movimento 21 dias\#30tododia\#21dias \#healthystyle\#vidasaudável\#corpoealma \#fitness (Usuária 4 do Instagram).

Esse caráter relacional de saúde presente nas postagens analisadas sugerem-nos pensar que, embora os indivíduos valham-se inicialmente dos argumentos propostos pelo programa, o que os fazem praticar determinadas ações são aquelas sensações e percepções ligadas ao campo da subjetividade. Para Canguilhem (2004, p. 63) a definição de saúde que inclui a referência da vida orgânica ao prazer e à dor, introduz sutilmente o conceito de corpo subjetivo.

Entendemos que o conceito de corpo subjetivo proposto por Canguilhem (2004) é importante para demarcar essa tensão que existe entre os sentidos orgânicos e relacionais que perpassam o ideário dos indivíduos que aderem à práticas do campo da promoção da saúde. A presença dessa tensão pode dar-nos pistas a respeito das outras formas de apropriação dos conselhos científicos (oferecidos por programas como o M21) por parte dos sujeitos. Nesse caso não há uma submissão total dos discursos calculistas pautados pelo risco e pela normalização, visto que, pela ótica dos indivíduos, por mais que eles possam dizer que aderiram a cuidados corporais e alimentares para perder peso, diminuir/aumentar taxas fisiológicas e antropométricas, o que mais parece importar a eles (ou importar tanto quanto) seriam essas sensações de bem estar, de "curtir a natureza", de sentirem-se mais leves de passarem mais tempo entre amigos e familiares. Contudo, é pertinente afirmar que o conceito de corpo subjetivo presente na obra de Canguilhem não se faz enquanto algo oposto ao saber científico, sendo que "[...] um não representa a alteridade radical do outro [...] o corpo subjetivo precisa destes saberes que Ihe indicam e sugerem uma série de artifícios úteis à sua sustentação" (Caponi, 2003, p. 60).

Neste jogo entre os distintos polos (saúde/ enfermidade, orgânico/subjetivo), percebe-se que cada vez mais os indivíduos poderão apresentar intencionalidades dinâmicas no que diz respeito às práticas cotidianas, mostrando variações entre os polos a depender das circunstâncias. A respeito disso, como já fora constatado em outras pesquisas realizadas com usuários e frequentadores de programas de saúde ou de espaços de lazer voltados para práticas de exercícios físicos na região metropolitana de Vitória (Silva Gomes, 2018; Herrera, 2018; Beccalli, 2012), os interesses apresentados pelos sujeitos para aderirem ao Movimento 21 dias são bastante difusos e híbridos, demonstrando que os discursos sobre a vida saudável 
podem adquirir distintas implicações e efeitos nos sujeitos que são interpeladas por eles. Algumas pessoas mostraram-se identificadas e instigadas com os discursos proferidos pelo programa, como podemos ver nas postagens que abordavam aspectos antropométricos do corpo aliados a cobranças morais sobre os próprios sujeitos. Por outro lado, também encontramos nas demais postagens outras preocupações relacionadas e vinculadas de certa forma à vida saudável, como no caso das pessoas que indicavam elementos gregários e relacionais ao referirem-se à suas práticas alimentares, familiares e de atividade física. Ainda há também aqueles indivíduos que mesclam esses dois movimentos, referindo-se tanto questões ascéticas quanto com sentimentos de fruição e bem-estar.

\section{CONSIDERAÇÕES FINAIS}

Podemos observar ao longo dessa investigação como diferentes sujeitos incorporaram em algum momento de suas vidas as premissas do Movimento 21 dias de maneira consentida e sem necessitarem de ações coercivas por parte do Governo do Estado. Interesses institucionais e individuais passaram a se relacionar entre si nesses casos, a ponto de as pessoas entenderam que teriam que fazer a "sua parte" para manter ou melhorar suas condições de vida nas formas que o programa indicou. Independente do quantitativo de indivíduos que aderiram ao programa é interessante perceber que esse tipo de mensagem passada pela campanha ainda encontra lugar na constituição de novas subjetividades e identidades.

As postagens analisadas nos indicam que em algum momento os indivíduos apoiaram-se nos conselhos do Movimento 21 dias, mostrando inclusive engajamento e implicação com seus desafios, registrando em imagens e textos os efeitos dos mesmos em suas vidas cotidianas. Também se notou que parte dos usuários utilizou o M21 como um conselheiro a mais para prover indicações sobre como ter uma vida mais saudável, sendo que em alguns momentos os próprios usuários se valeram dessa série de conselhos para constituíremse em novos conselheiros do estilo de vida saudável, indicando receitas e exercícios físicos em suas postagens nas redes sociais. A autocobrança, o selftracking e o senso de responsabilidade consigo mesmo foram marcas recorrentes nas "confissões" escritas e compartilhadas pelos sujeitos, inclusive demonstrando sensações e sentimentos como fracasso, vergonha e culpa por não atingirem seus objetivos e de superação e determinação quando os mesmos eram alcançados, corroborando em partes, pelo menos discursivamente, com os pressupostos lançados pelos enunciados do M21. Com isso, é preciso enfatizar que os sentidos atribuídos pelos sujeitos ao discurso da vida saudável são provisórios e os efeitos de tal discurso sobre a constituição da subjetividade dos indivíduos não demonstram uniformidade.

Podemos dizer que essa consideração se deve ao fato de que os efeitos da apropriação de um determinado discurso variam de acordo com os modos pelos quais as pessoas vão constituindo suas experiências, levando em conta as condições e as histórias de vida de cada uma. Ao longo desta investigação, interpretamos que há possibilidades de múltiplas leituras, interpretações e recepções dos conteúdos de um dispositivo de saúde móvel que visa justamente à homogeneização, à normalização e ao governo das condutas.

\section{REFERÊNCIAS}

Abib LT. O governo de si e dos outros em dispositivos de saúde móvel: reflexões sobre o "Movimento 21 dias por uma vida mais saudável" [Tese] Vitória: Universidade Federal do Espírito Santo; 2019.

Abib LT, Gomes IM. A educação e o governamento dos corpos na atualidade: uma análise do "Movimento 21 dias por uma vida mais saudável". Saúde e Sociedade 2019; 28(4): 229-242.

Beccalli MB. Mais que atividade física: os usos e entendimentos da saúde entre usuários do serviço de orientação ao exercício da prefeitura municipal de Vitória [Dissertação] Vitória: Universidade Federal do Espírito Santo; 2012.

Brasil MS. Política Nacional de Promoção da Saúde. Portaria ${ }^{\circ}$ 687 MS/GM, de 30 de março de 2006. Aprova a Política de Promoção da Saúde. Diário Oficial da União 2006; 31 mar.

Canguilhem G. Escritos sobre la medicina. Buenos Aires: Amorrotu; 2004.

Caponi S. A saúde como abertura ao risco. In: Czeresnia D, Freitas CM (Orgs.). Promoção da saúde (conceitos, reflexões, tendências). Rio de Janeiro: FIOCRUZ; 2003. 55-78.

Castiel LD, Moraes DR, Paula IJ. Terapeuticalização e os dilemas preemptivistas na esfera da saúde pública inidividualizada. Saúde e Sociedade 2016; 25(1): 96-107.

Fotopoulou A, O'Riordan K. Training to self-care: fitness tracking, biopedagogy and the healthy consumer. Health Sociology Review 2016; 26(1): 54-68.

Foucault M. Segurança, território, população. São Paulo: Martins Fontes; 2008.

Fraga $A B$. Exercício da informação: governo dos corpos no mercado da vida ativa. Campinas: Autores Associados; 2006.

Fragoso S, Recuero R, Amaral A. Métodos de pesquisa para internet. Porto Alegre: Sulina; 2011.

Fullagar S, Rich E, Francombe-Webb J, Maturo A. Digital Ecologies of Youth Mental Health: Apps, Therapeutic Publics and Pedagogy as Affective Arrangements. Society and Science 2017; 6(135): 1-14.

Gomes IM. Conselheiros Modernos: propostas para a educação do indivíduo saudável [Tese] Florianópolis: Universidade Federal de Santa Catarina; 2008. 
Goodyear VA. Social media, apps and wearable technologies: navigating ethical dilemas and procedures. Qualitiative Research in Sport, Exercise and Health 2017; 9(3): 285-302.

Han BC. Psicopolítica. Barcelona: Herder; 2014.

Herrera REG. Corpos e Ambivalências: uma breve etnografia na praia de Camburi [Dissertação] Vitória: Universidade Federal do Espírito Santo; 2018.

Lupton, D. M-health and health promotion: the digital cyborg and surveillance society. Social theory e health 2012; 10(3): 229-244.

Lupton D, Thomas GM. Playing pregnancy: the ludification and gamification of expectante motherhood. M/C Journal. 2015; 18(5). Disponível em http://journal.media-culture. org.au/index.php/mcjournal/article/view/1012 Acesso em 25 de outubro de 2019.

Lupton D, editora. Self-tracking, health and medicine: sociological perspectives. Abingdon: Routledge; 2018.

Matravolgyi J. Plataformas para saúde já têm 165 mil aplicativos. Valor Econômico, 21 de nov. de 2016. Disponível em http://www.i9access.com.br/noticias_arquivos/valor_ plataformas_2016.pdf Acesso em 30 de novembro de 2018.

Ravettino AJ. El estilo de vida light: hábitos y patrones de consumo. Revista científica de UCES. 2008; 12(1): 103-117.
Riley WT et al. Health behavior models in the age of mobile interventions: are our theories up to the task? Translational behavior medicine. 2011; 1(1): 53-71.

Rocha TAH et al. Saúde Móvel: novas perspectivas para a oferta de serviços em saúde. Epidemiologia e Serviços de Saúde. 2016; 25(1): 159-170.

Rose N. Governando a alma: a formação do eu privado. In: Silva TT (Org.). Liberdades reguladas: a pedagogia construtivista e outras formas de governo do eu. Petrópolis: Vozes, 1998; 30-45.

Silva Gomes LR. O yoga no Serviço de Orientação ao Exercício (SOE) em Vitória: ambivalências acerca dos significados atribuídos a uma prática corporal oriental [Tese] Vitória: Universidade Federal do Espírito Santo; 2018.

Silva T, Stabile M. Monitoramento e pesquisa em mídias sociais. São Paulo: Uva Limão; 2016.

Who. mHealth: New horizons for health through mobile technologies: second global survey on eHealth. Disponível em <http://www.who.int/goe/publications/goe_mhealth_ web.pdf > Acessado em 26 de abril de 2018.

Wright J, Hardwood V, editoras. Biopolitics and the "obesity epidemic": governing bodies. Londres: Routledge; 2009. 